Research Article

\title{
Permanence and Existence of Periodic Solutions for Nicholson's Blowflies Model with Feedback Control and Delay on Time Scales
}

\author{
Lin-Lin Wang $(\mathbb{D}$ and Yong-Hong Fan $(\mathbb{D})$ \\ School of Mathematics and Statistics Science, Ludong University, Yantai, Shandong 264025, China \\ Correspondence should be addressed to Yong-Hong Fan; fanyh_1993@sina.com
}

Received 21 April 2018; Accepted 26 July 2018; Published 8 August 2018

Academic Editor: Luca Pancioni

Copyright (C) 2018 Lin-Lin Wang and Yong-Hong Fan. This is an open access article distributed under the Creative Commons Attribution License, which permits unrestricted use, distribution, and reproduction in any medium, provided the original work is properly cited.

Sufficient conditions are established for the permanence in a delayed Nicholson's blowflies model with feedback control on time scales. Our investigation confirms that the bounded feedback terms do not have any influence on the permanence of this system.

\section{Introduction}

In 2008, we considered the following discrete Nicholson's blowflies model with feedback control (see [1]):

$$
\begin{aligned}
& x(n+1)=x(n) \exp \{-\delta(n)+p(n) \exp \{-\alpha(n) x(n)\} \\
& \quad-c(n) U(n)\}, \\
& \Delta U(n)=-a(n) U(n)+b(n) x(n-m),
\end{aligned}
$$

where $\delta, \alpha, p, b$, and $c: \mathbb{Z}$ (integer number set) $\longrightarrow \mathbb{R}^{+}$ (nonnegative real number set) are all bounded sequences, $a: \mathbb{Z} \longrightarrow(0,1)$ is also a bounded sequence and inf $a>0$, $m$ is a nonnegative integer, and $\Delta$ is the first-order former difference operator and obtained the following.

Theorem 1. Assume that

$$
\inf _{n \in[0,+\infty) \cap \mathbb{Z}}(p(n)-\delta(n))>0
$$

hold true; then system (1) is permanent.

The continuous and discrete systems always appear separately, until in 1988, the theory of time scales, which has recently received much attention, was initiated by Hilger [2] in his Ph.D. thesis to unify both difference and differential calculus in a consistent way. Since then many authors have investigated the dynamic equations on time scales (see [3-6]). This theory provides a powerful tool for applications to economics, population models, quantum physics, among others. In fact, the progressive field of dynamic equations on time scales contains, links, and extends the classical theory of differential and difference equations.

For the origin of mathematical model for Nicholson's blowflies, one can see [7]. For further study on equations with feedback control, we refer to [8-14] and references therein. And for investigation on delay differential equations, we refer to $[15,16]$ and so on.

In this paper, we will discuss the permanence of the following system:

$$
\begin{aligned}
x^{\Delta}(t)= & -\delta(t)+p(t) \exp \{-\alpha(t) \exp \{x(t-\tau(t))\}\} \\
& -c(t) y(t-\theta(t)), \\
y^{\Delta}(t)= & -a(t) y(t)+b(t) \exp \{x(t-\eta(t))\},
\end{aligned}
$$

on time scales $\mathbb{T}$, where $\Delta$ stands for the delta-derivative and

$$
1-\mu(t) a(t)>0,
$$


in which $\mu(t)=\sigma(t)-t$ and $\sigma(t)$ is the forward jump operator on $\mathbb{T}$. To make (3) meaningful, we suppose that, for any $t \in \mathbb{T}$,

$$
\begin{aligned}
& t-\tau(t) \in \mathbb{T}, \\
& t-\theta(t) \in \mathbb{T}, \\
& t-\eta(t) \in \mathbb{T} .
\end{aligned}
$$

We assume that sup $\mathbb{T}=\infty$, and, without loss of generality, suppose $0 \in \mathbb{T}$. In view of the biological significance, we also assume that the coefficient functions $\delta, \alpha, p, b, c$, $\tau, \theta$, and $\eta: \mathbb{T} \longrightarrow \mathbb{R}^{+}$are all bounded rd-continuous and inf $_{t \in \mathbb{T}} a>0$ (the definition of rd-continuous function will be given in Section 2).

When $\mathbb{T}=\mathbb{R}$, let $N(t)=\exp \{x(t)\}, U(t)=y(t)$, and then (3) can be rewritten as

$$
\begin{aligned}
N^{\prime}(t)= & -\delta(t) N(t) \\
& +p(t) N(t) \exp \{-\alpha(t) N(t-\tau(t))\} \\
& -c(t) U(t-\theta(t)), \\
U^{\prime}(t)= & -a(t) U(t)+b(t) N(t-\eta(t)) .
\end{aligned}
$$

When $\mathbb{T}=\mathbb{Z}$, if we let $N(t)=\exp \{x(t)\}$, then (3) can be rewritten as

$$
\begin{aligned}
& N(n+1)=N(n) \exp \{-\delta(n) \\
& \quad+p(n) \exp \{-\alpha(n) x(n-\tau(n))\} \\
& \quad-c(n) U(n-\theta(n))\}, \\
& \Delta U(n)=-a(n) U(n)+b(n) x(n-\eta(n)) .
\end{aligned}
$$

Obviously, (7) includes (1).

In what follows we shall use the notations

$$
\begin{gathered}
f^{u}=\sup _{t \in \mathbb{T}} f(t), \\
f^{l}=\inf _{t \in \mathbb{T}} f(t),
\end{gathered}
$$

where $f$ is a bounded rd-continuous function in $\mathbb{T}$. Throughout this paper, we assume that

$$
\begin{aligned}
& a^{l}>0, \\
& \delta^{l}>0 .
\end{aligned}
$$

In this case, for any $t \in \mathbb{T}$,

$$
\mu(t) \leq\left(\frac{1}{a(t)}\right)^{u}:=L .
$$

\section{Preliminary}

Before giving our main result, first we list some basic properties about time scales which could be found in $([2,17$, 18]).
Definition 2. A time scale is an arbitrary nonempty closed subset $\mathbb{T}$ of the real number $\mathbb{R}$.

Definition 3. For $t \in \mathbb{T}$ we define the forward jump operator $\sigma: \mathbb{T} \longrightarrow \mathbb{T}$ and the backward jump operator $\rho: \mathbb{T} \longrightarrow \mathbb{T}$, by

$$
\begin{aligned}
& \sigma(t):=\inf \{s \in \mathbb{T}: s>t\}, \\
& \rho(t):=\sup \{s \in \mathbb{T}: s<t\},
\end{aligned}
$$

respectively.

Definition 4. Define the interval $[a, b]$ in $\mathbb{T}$ by

$$
[a, b]:=\{t \in \mathbb{T}: a \leq t \leq b\} .
$$

Other types of intervals are defined similarly.

Definition 5 (Definition 1.58 in [18], P22). A function $f$ : $\mathbb{T} \longrightarrow \mathbb{R}$ is called $\mathrm{rd}$-continuous provided that it is continuous at right-dense points in $\mathbb{T}$ and its left-sided limits exist (finite) at left-dense points in $\mathbb{T}$. The set of rd-continuous functions $f: \mathbb{T} \longrightarrow \mathbb{R}$ is denoted by

$$
C_{r d}=C_{r d}(\mathbb{T})=C_{r d}(\mathbb{T}, \mathbb{R})
$$

Definition 6. Assume $f: \mathbb{T} \longrightarrow \mathbb{R}$ and let $t \in \mathbb{T}^{k}$, where

$$
\mathbb{T}^{k}= \begin{cases}\mathbb{T} \backslash(\rho(\sup \mathbb{\mathbb { V }}), \sup \mathbb{\mathbb { T }}], & \text { if } \sup \mathbb{T}<\infty, \\ \mathbb{T}, & \text { if } \sup \mathbb{T}=\infty .\end{cases}
$$

Then we define $f^{\Delta}(t)$ to be the number (provided it exists) with property that given any $\varepsilon>0$, there is a neighborhood $U$ of $t$ such that

$$
\left|[f(\sigma(t))-f(s)]-f^{\Delta}(t)[\sigma(t)-s]\right| \leq \varepsilon|\sigma(t)-s|,
$$

for all $s \in U$. We call $f^{\Delta}(t)$ the delta (or Hilger) derivative of $f(t)$ and it turns out that $f^{\Delta}$ is the usual derivative if $\mathbb{T}=\mathbb{R}$ and is the usual forward difference operator if $\mathbb{T}=\mathbb{Z}$.

Lemma 7 (Theorem 1.76 in [18], P28). If $f^{\Delta} \geq 0$, then $f$ is increasing.

Definition 8 (Definition 2.25 in [18], P58). We say that a function $p: \mathbb{T} \longrightarrow \mathbb{R}$ is regressive provided that

$$
1+\mu(t) p(t) \neq 0 \quad \text { for all } t \in \mathbb{T}^{k}
$$

holds. The set of all regressive and rd-continuous functions $f: \mathbb{T} \longrightarrow \mathbb{R}$ will be denoted by

$$
\mathfrak{R}=\mathfrak{R}(\mathbb{T})=\mathfrak{R}(\mathbb{\mathbb { T }}, \mathbb{R}) .
$$

Definition 9. If $p \in \Re$, we define the exponential function by

$$
e_{p}(t, s)=\exp \left(\int_{s}^{t} \xi_{\mu(\tau)}(p(\tau)) \Delta \tau\right), \quad \text { for } s, t \in \mathbb{T},
$$

where the cylinder transformation $\xi_{h}(z)=(1 / h) \log (1+z h)$, for $h>0$. 
For $h=0$, we define $\xi_{0}(z)=z$. In this case, $\mathbb{T}=\mathbb{R}, \mu(t)=$ 0 , and $e_{p}(t, s)=\exp \left(\int_{s}^{t} p(\tau) \Delta \tau\right)$.

When $\mathbb{T}=h \mathbb{Z}=\{h k: k \in \mathbb{Z}\}$, we know that $\mu(h k)=$ $h(k+1)-h k=h>0$, and then for $s<t$,

$$
\begin{aligned}
e_{p}(t, s) & =\exp \left\{\int_{s}^{t} \frac{1}{h} \log _{e}(1-h a(\tau)) \Delta \tau\right\} \\
& =\exp \left\{\sum_{k=s / h}^{t / h-1} \log _{e}(1-h a(h k))\right\} \\
& =\prod_{k=s / h}^{t / h-1}\{1-h a(h k)\} .
\end{aligned}
$$

Definition 10. If $p \in \Re$, the function $\Theta(p)$ is defined by

$$
\ominus(p)(t)=-\frac{p(t)}{1+\mu(t) p(t)}, \quad \text { for } t \in \mathbb{T}^{k} .
$$

Lemma 11. Suppose $p \in \mathfrak{R}$; then

$$
\begin{aligned}
& \text { (1) } \ominus(\ominus(p))=p ; \\
& \text { (2) } e_{p}(\sigma(t), s)=(1+\mu(t) p(t)) e_{p}(t, s) \text {; } \\
& \text { (3) } 1 / e_{p}(t, s)=e_{\ominus p}(t, s) \text {; } \\
& \text { (4) }\left(1 / e_{p}(\cdot, s)\right)^{\Delta}=-p(\cdot) / e_{p}^{\sigma}(\cdot, s) \text {; } \\
& \text { (5) }\left(e_{p}(\cdot, s)\right)^{\Delta}=p(\cdot) e_{p}(\cdot, s) .
\end{aligned}
$$

Lemma 12 (Theorem 2.44 in [18], P66). Assume that $p \in \Re$ and $t_{0} \in \mathbb{T}$. If $1+\mu(t) p(t)>0$ on $\mathbb{T}^{k}$, then $e_{p}\left(t, t_{0}\right)>0$ for all $t \in \mathbb{T}$.

Lemma 13 (Theorem 2.77 in [18], P77). Suppose $p \in \Re$ and $f: \mathbb{T} \longrightarrow \mathbb{R}$ is rd-continuous. Let $t_{0} \in \mathbb{T}$ and $y_{0} \in \mathbb{R}$. The unique solution of the initial value problem

$$
\begin{aligned}
y^{\Delta} & =p(t) y+f(t), \\
y\left(t_{0}\right) & =y_{0}
\end{aligned}
$$

is given by

$$
y(t)=e_{p}\left(t, t_{0}\right) y_{0}+\int_{t_{0}}^{t} e_{p}(t, \sigma(s)) f(s) \Delta s .
$$

In order to give our main result, we also need to establish the following definitions and lemmas. The first definition is the generalized version of the semicycle in discrete situation [19].

Definition 14. Let $m$ be a constant and $f: \mathbb{T} \longrightarrow \mathbb{R}$; a positive semicycle relative to $m$ of $f$ is defined as follows, as it consists of a "string" of terms:

$$
\{f(t), t \in[s, t], s, t \in \mathbb{T}\},
$$

all greater than or equal to $m$, and a negative semicycle relative to $m$ of $f$ is defined as follows, as it is a "string" of terms:

$$
\{f(t), t \in[s, t], s, t \in \mathbb{T}\},
$$

all less than or equal to $m$.
The following two lemmas could be found in [20].

Lemma 15. Assume that $A, B>0$ and $x_{1}(0)=x_{0}>0$; furthermore suppose that

(1)

$$
x_{1}^{\Delta}(t) \leq B-A \exp \left\{x_{1}(t)\right\}, \quad \text { for } t \geq 0,
$$

and then

$$
\lim _{t \rightarrow \infty} \sup x_{1}(t) \leq B L+\ln \frac{B}{A},
$$

$$
x_{1}^{\Delta}(t) \geq B-A \exp \left\{x_{1}(t)\right\}, \text { for } t \geq 0,
$$

and there exists a constant $M>0$, such that $\lim _{t \rightarrow \infty} \sup x_{1}(t)<M$; then

$$
\lim _{t \longrightarrow \infty} \inf x_{1}(t) \geq(B-A \exp \{M\}) L+\ln \frac{B}{A} .
$$

Lemma 16. Assume that $C(t), D(t)>0$ are bounded $r d$ continuous functions and $C^{l}>0$; furthermore suppose that the $\Delta$-differentiable function $y_{1}(t)$ satisfies

(1)

$$
y_{1}^{\Delta}(t)+C(t) y_{1}(t) \leq D(t), \quad \text { for } t \geq T_{0},
$$

and $D(t)$ is $\Delta$-differentiable; then there exists a constant $T_{2}>$ $T_{0}$, such that for $t>T_{2}$, we have

$$
y_{1}(t) \leq y_{1}\left(T_{2}\right) e_{(-C)}\left(t, T_{2}\right)+\frac{D(t)}{C^{l}} .
$$

Especially, if $D(t)$ is bounded above ultimately with respect to $K_{1}$, then

$$
\lim _{t \rightarrow \infty} \sup y_{1}(t) \leq \frac{K_{1}}{C^{l}}
$$

(2)

$$
y_{1}^{\Delta}(t)+C(t) y_{1}(t) \geq D(t), \quad \text { for } t \geq T_{0},
$$

and there exists a constant $T_{3}>T_{0}$, such that, for $t>T_{3}$, we have

$$
y_{1}(t) \geq\left(y_{1}\left(T_{3}\right)-\frac{D\left(T_{3}\right)}{C^{u}}\right) e_{(-C)}\left(t, T_{3}\right)+\frac{D\left(T_{3}\right)}{C^{u}} .
$$

Especially, if $D(t)$ is bounded below ultimately with respect to $k_{1}$, then

$$
\lim _{t \rightarrow \infty} \inf y_{1}(t) \geq \frac{k_{1}}{C^{u}}
$$

Before giving our main result, we list the definition of uniform ultimate boundedness. 
Definition 17. Solutions for system (3) are said to be uniformly ultimate bounded if there exist two constants $\lambda_{1}$ and $\lambda_{2}$ such that, for any initial condition

$$
\begin{aligned}
(\phi(t), \psi(t))^{T} \in C_{r d}\left([-\lambda, 0), \mathbb{R} \times \mathbb{R}^{+}\right), & \\
\lambda & =\max \left\{\tau^{u}, \eta^{u}, \theta^{u}\right\},
\end{aligned}
$$

we have

$$
\begin{aligned}
& \lambda_{1} \leq \lim _{t \rightarrow \infty} \inf x(t) \leq \lim _{t \rightarrow \infty} \sup x(t) \leq \lambda_{2}, \\
& \lambda_{1} \leq \lim _{t \rightarrow \infty} \inf y(t) \leq \lim _{t \rightarrow \infty} \sup y(t) \leq \lambda_{2},
\end{aligned}
$$

where $\lambda_{1}$ and $\lambda_{2}$ are independent of $(\phi(t), \psi(t))^{T}$.

\section{Main Results}

First, we give a lemma which will be useful for our further discussion.

Lemma 18. Let $(x(t), y(t))$ be any solution of system (3) with initial condition (35); then

$$
\begin{aligned}
\exp \{x(t)\} & >0, \\
y(t) & >0
\end{aligned}
$$

for all $t \in \mathbb{T}$.

Proof. The exponential form $\exp \{x(t)\}$ ensures that $\exp \{x(t)\}>0$ for all $t \in \mathbb{T}$. Now we consider the second equation of system (3); by Lemma 13 and (4), we have

$$
\begin{aligned}
y(t)= & e_{-a}(t, 0) y_{0} \\
& +\int_{0}^{t} e_{-a}(t, \sigma(s)) b(s) \exp \{x(s-\eta(s))\} \Delta s \\
> & 0 \quad \text { for } t \geq 0 .
\end{aligned}
$$

The proof is complete.

In the sequel, we assume that

$$
(p(n)-\delta(n))^{l}>0 .
$$

Theorem 19. Assume that (39) holds true; then system (3) is uniformly ultimate bounded.

We now prove the following result before proving Theorem 19. In fact, the two theorems are equivalent to each other.

Theorem 20. Assume that (39) holds true; then there exist two positive constants $\lambda_{1}$ and $\lambda_{2}$ such that

$$
\begin{aligned}
\lambda_{1} & \leq \lim _{t \rightarrow \infty} \inf \exp \{x(t)\} \leq \lim _{t \rightarrow \infty} \sup \exp \{x(t)\} \\
& \leq \lambda_{2},
\end{aligned}
$$

and

$$
\lambda_{1} \leq \lim _{t \rightarrow \infty} \inf y(t) \leq \lim _{t \rightarrow \infty} \sup y(t) \leq \lambda_{2}
$$

for any solutions $(x(t), y(t))$ of (3) with initial condition (35).
Proof. We divided the proof into four claims.

Claim 1. There exists a positive constant $K_{1}$ such that

$$
\lim _{t \rightarrow \infty} \sup x(t) \leq K_{1} \text {, }
$$

where $K_{1}$ can be chosen as

$$
K_{1}=\frac{\exp \left\{\left(p^{u}-\delta^{l}\right) \tau^{u}\right\}}{\alpha^{l}} \ln \frac{p^{u}}{\delta^{l}}+\left(p^{u}-\delta^{l}\right) l .
$$

Proof. From the first equation of system (3) and the positivity of $y(t)$, we have

$$
\begin{aligned}
x^{\Delta}(t) & \leq-\delta(t)+p(t) \exp \{-\alpha(t) \exp \{x(t-\tau(t))\}\} \\
& \leq-\delta^{l}+p^{u} \exp \left\{-\alpha^{l} \exp \{x(t-\tau(t))\}\right\} .
\end{aligned}
$$

From the first equation of (3), we have

$$
x^{\Delta}(t) \leq p^{u}-\delta^{l}, \quad \text { for all } t \in \mathbb{T},
$$

thus

$$
x(t)-x(t-\tau(t)) \leq\left(p^{u}-\delta^{l}\right) \tau(t) \leq\left(p^{u}-\delta^{l}\right) \tau^{u},
$$

which implies that

$$
\exp \{x(t-\tau(t))\} \geq \exp \left\{-\left(p^{u}-\delta^{l}\right) \tau^{u}\right\} \exp \{x(t)\},
$$

and by (44), we have

$$
\begin{aligned}
x^{\Delta}(t) & \\
\leq & -\delta^{l} \\
& +p^{u} \exp \left\{-\alpha^{l} \exp \left\{-\left(p^{u}-\delta^{l}\right) \tau^{u}\right\} \exp \{x(t)\}\right\},
\end{aligned}
$$

and for simplicity, we set

$$
A_{1}=\alpha^{l} \exp \left\{-\left(p^{u}-\delta^{l}\right) \tau^{u}\right\}
$$

obviously, $A_{1}>0$. In the following, we divided the proof into three cases:

(1) There exists some $N_{1} \in \mathbb{T}$ such that $x(t) \leq$ $\left(1 / A_{1}\right) \ln \left(p^{u} / \delta^{l}\right)$ for all $t>N_{1}$, and then we have

$$
\lim _{t \rightarrow \infty} \sup x(t) \leq \frac{1}{A_{1}} \ln \frac{p^{u}}{\delta^{l}}<K_{1} .
$$

(2) There exists some $N_{2} \in \mathbb{T}$ such that $x(t)>$ $\left(1 / A_{1}\right) \ln \left(p^{u} / \delta^{l}\right)$ for all $t>N_{2}$, and then from (48), we have

$$
x^{\Delta}(t) \leq 0 \text { for } t>N_{2}+\tau^{u}
$$

which implies that $\lim _{t \longrightarrow+\infty} x(t)$ exists and if we denote $\lim _{t \rightarrow+\infty} x(t)=\lambda$, and then

$$
\lambda \geq \frac{1}{A_{1}} \ln \frac{p^{u}}{\delta^{l}}
$$


and thus (48) shows that

$$
\left\{-\delta^{l}+p^{u} \exp \left\{-A_{1} \lambda\right\}\right\} \geq 0
$$

that is,

$$
\lambda \leq \frac{1}{A_{1}} \ln \frac{p^{u}}{\delta^{l}}
$$

while from (52) and (54), we get

$$
\lambda=\frac{1}{A_{1}} \ln \frac{p^{u}}{\delta^{l}}
$$

therefore

$$
\lim _{t \rightarrow \infty} \sup x(t)=\frac{1}{A_{1}} \ln \frac{p^{u}}{\delta^{l}}<K_{1} .
$$

(3) For simplicity, set $\beta=\left(1 / A_{1}\right) \ln \left(p^{u} / \delta^{l}\right)$. Assume that $x(t)$ oscillates about $\beta$. By (48), we know that $u(t) \geq \beta$ implies that $x^{\Delta}(t) \leq 0$. Thus, by Lemma 7, if we let $\left\{x\left(t_{l}\right)\right\}$ be the first element of a $l$ th positive semicycle relative to $\beta$ of $\{x(t)\}$, then

$$
\lim _{t \rightarrow \infty} \sup x(t)=\lim _{t \rightarrow \infty} \sup x\left(t_{l}\right) .
$$

Now we divided the proof into two cases: $t_{l}$ is left-dense and $t_{l}$ is left-scattered. If the former holds, then

$$
x\left(t_{l}\right)=\beta .
$$

If the latter holds, then $x\left(\rho\left(t_{l}\right)\right) \leq \beta$, by (45), we have

$$
\begin{aligned}
\int_{\rho\left(t_{l}\right)}^{t_{l}} x^{\Delta}(s) \Delta s & \leq \int_{\rho\left(t_{l}\right)}^{t_{l}}\left(p^{u}-\delta^{l}\right) \Delta s \\
& =\left(p^{u}-\delta^{l}\right)\left(t_{l}-\rho\left(t_{l}\right)\right) \leq\left(p^{u}-\delta^{l}\right) L,
\end{aligned}
$$

and therefore

$$
x\left(t_{l}\right) \leq x\left(\rho\left(t_{l}\right)\right)+\left(p^{u}-\delta^{l}\right) l \leq \beta+\left(p^{u}-\delta^{l}\right) L,
$$

while from (58) and (60), we have

$$
\lim _{t \rightarrow \infty} \sup x\left(t_{l}\right) \leq \beta+\left(p^{u}-\delta^{l}\right) L=K_{1} .
$$

By (50), (56), and (61), we complete the proof of Claim 1.

Claim 2. There exists a positive constant $K_{2}$ such that

$$
\lim _{t \rightarrow \infty} \sup y(t) \leq K_{2} \text {. }
$$

Proof. From (42), we know that, for any arbitrary positive number $\varepsilon$, there exists an $N_{3}$ such that $x(t) \leq K_{1}+\varepsilon$ for all $t>N_{3}$, and then from the second equation of system (3), we have

$$
y^{\Delta}(t) \leq-a(t) y(t)+b^{u} \exp \left\{K_{1}+\varepsilon\right\},
$$

by Lemma $16(1)$,

$$
\lim _{t \rightarrow \infty} \sup y(t) \leq \frac{b^{u} \exp \left\{K_{1}+\varepsilon\right\}}{a^{l}} .
$$

Let $\varepsilon \longrightarrow 0$, and then we can obtain

$$
\lim _{t \rightarrow \infty} \sup y(t) \leq \frac{b^{u} \exp \left\{K_{1}\right\}}{a^{l}}:=K_{2} .
$$

The proof of Claim 2 is complete.

Claim 3. There exists a constant $k_{1}$ such that

$$
\lim _{t \rightarrow \infty} \inf x(t) \geq k_{1}
$$
have

By Claims 1 and 2 and the first equation of system (3), we

$$
x^{\Delta}(t) \geq-\delta^{u}+p^{l} \exp \left\{-\alpha^{u} \exp \left\{K_{1}\right\}\right\}-c^{u} K_{2} \geq-A_{2},
$$

for $t$ sufficiently large, where $A_{2}>0$ is a constant. Then

$$
\begin{aligned}
& x(t)-x(t-\eta(t)) \geq-A_{2} \eta(t) \geq-A_{2} \eta^{u}, \\
& x(t)-x(t-\tau(t)) \geq-A_{2} \tau(t) \geq-A_{2} \tau^{u} .
\end{aligned}
$$

From the second equation of system (3), we have

$$
y^{\Delta}(t) \leq-a(t) y(t)+b^{u} \exp \left\{A_{2} \tau^{u}\right\} \exp \{x(t)\},
$$

and then Lemma 16 (1) implies that, for any $t>T_{2}$,

$$
\begin{aligned}
y(t) \leq & y\left(T_{2}\right) e_{(-a)}\left(t, T_{2}\right) \\
& +\frac{b^{u} \exp \left\{A_{2} \tau^{u}\right\} \exp \{x(t)\}}{a^{l}},
\end{aligned}
$$

noting that

$$
y\left(T_{2}\right) e_{(-a)}\left(t, T_{2}\right) \longrightarrow 0, \quad \text { as } t \longrightarrow+\infty
$$

and hence there exists a positive integer $K>T_{2}$ such that, for any solution $(x(t), y(t))$ of system (3), $y\left(T_{2}\right) c(t) e_{(-a)}\left(t, T_{2}\right)<$ $\gamma / 2$, as $t>K$, where $\gamma=\inf _{t \in \mathbb{T}}(p(t)-\delta(t))$. Fixing $K$, we get

$$
y(t) \leq y\left(T_{2}\right) e_{(-a)}\left(t, T_{2}\right)+\frac{b^{u} \exp \left\{A_{2} \tau^{u}\right\} \exp \{x(t)\}}{a^{l}}
$$

Set

$$
M_{1}=\frac{b^{u} \exp \left\{A_{2} \tau^{u}\right\}}{a^{l}}
$$

and then

$$
y(t) \leq y\left(T_{2}\right) e_{(-a)}\left(t, T_{2}\right)+M_{1} \exp \{x(t)\} .
$$


Notice that $e^{-x} \geq 1-x$, for $x>0$, and then from the first equation of system (3), we have

$$
\begin{aligned}
x^{\Delta}(t) \geq & -\delta(t)+p(t)(1-\alpha(t) \exp \{x(t-\tau(t))\}) \\
& -c(t) y(t-\theta(t)) \\
\geq & p(t)-\delta(t) \\
& -(p(t) \alpha(t))^{u} \exp \left\{A_{2} \tau^{u}\right\} \exp \{x(t)\} \\
& -c(t) y\left(T_{2}\right) e_{(-a)}\left(t, T_{2}\right) \\
& -M_{1} c(t) \exp \{x(t)\}
\end{aligned}
$$

for $t>K$. According to the choosing of $K$, we have

$$
\begin{aligned}
x^{\Delta}(t) & \\
\geq & \frac{\gamma}{2} \\
& -\left((p(t) \alpha(t))^{u} \exp \left\{A_{2} \tau^{u}\right\}+M_{1} c^{u}\right) \exp \{x(t)\} \\
& \quad \text { for } t>K .
\end{aligned}
$$

By Lemma 15 (2), we have

$$
\lim _{t \rightarrow+\infty} \inf x(t) \geq\left(B-A \exp \left\{K_{1}\right\}\right) L+\ln \frac{B}{A},
$$

where

$$
B=\left((p(t) \alpha(t))^{u} \exp \left\{A_{2} \tau^{u}\right\}+M_{1} c^{u}\right), \quad A=\frac{\gamma}{2},
$$

and choosing

$$
k_{1}=\left(B-A \exp \left\{K_{1}\right\}\right) L+\ln \frac{B}{A},
$$

this shows that the conclusion holds true.

Claim 4. There exists a positive constant $k_{2}$ such that

$$
\lim _{t \longrightarrow+\infty} \inf y(t) \geq k_{2} \text {. }
$$

Notice that (66) implies that there exists an $N_{4}$ such that $x(t) \geq k_{1} / 2$ for all $t>N_{4}$, and then from the second equation of system (3), we have

$$
y^{\Delta}(t) \geq-a(t) y(t)+b^{l} \exp \left\{\frac{k_{1}}{2}\right\}
$$

$$
\text { for } t>N_{4}+\eta^{u}
$$

by Lemma $16(2)$,

$$
\lim _{t \longrightarrow+\infty} \inf y(t) \geq \frac{b^{l} \exp \left\{k_{1} / 2\right\}}{a^{u}}:=k_{2} .
$$

The proof of Claim 4 is complete.

Choose

$$
\begin{aligned}
& \lambda_{1}=\min \left\{\exp \left\{k_{1}\right\}, k_{2}\right\}, \\
& \lambda_{2}=\max \left\{\exp \left\{K_{1}\right\}, K_{2}\right\},
\end{aligned}
$$

obviously,

$$
\lambda_{i}>0, \quad i=1,2 \text {. }
$$

And

$$
\begin{aligned}
& \lambda_{1} \leq \lim _{t \longrightarrow \infty} \inf \exp \{x(t)\} \leq \lim _{t \rightarrow \infty} \sup \exp \{x(t)\} \leq \lambda_{2}, \\
& \lambda_{1} \leq \lim _{t \longrightarrow \infty} \inf y(k) \leq \lim _{t \rightarrow \infty} \sup y(t) \leq \lambda_{2} .
\end{aligned}
$$

Thus we complete the proof of Theorem 20 .

Theorem 21. If the coefficient functions $\delta, \alpha, p, b, c, \tau, \theta$, and $\eta: \mathbb{T} \longrightarrow \mathbb{R}^{+}$are all $\omega$-periodic $r d$-continuous functions and also satisfy $\bar{p}>\bar{\delta}$ and (9), then system (3) has at least an $\omega$-periodic solution with strictly positive for its second component.

Corollary 22. If all the conditions in Theorem 21 hold, then (6) or (7) has at least a positive $\omega$-periodic solution.

\section{Data Availability}

No data were used to support this study.

\section{Conflicts of Interest}

The authors declare that there are no conflicts of interest regarding the publication of this paper.

\section{Authors' Contributions}

All authors contributed equally to this work. All authors read and approved the final manuscript.

\section{Acknowledgments}

This work is supported by NSF of China (11201213, 11371183), NSF of Shandong Province (ZR2015AM026), and the Project of Shandong Provincial Higher Educational Science and Technology (J15LI07).

\section{References}

[1] L.-L. Wang and Y.-H. Fan, "Permanence for a discrete Nicholson's blowflies model with feedback control and delay," International Journal of Biomathematics, vol. 1, no. 4, pp. 433-442, 2008.

[2] S. Hilger, Ein Mab kettenkalkül mit Anwendung auf Zentrumsmannig faltigkeiten [Ph.D. thesis], Universität of Würzburg, 1988.

[3] R. P. Agarwal, M. Bohner, and D. O’Regan, “Time scale boundary value problems on infinite intervals," Journal of Computational and Applied Mathematics, vol. 141, no. 1-2, pp. 27-34, 2002.

[4] C. Wang and R. P. Agarwal, "Uniformly rd-piecewise almost periodic functions with applications to the analysis of impulsive D-dynamic system on time scales," Applied Mathematics and Computation, vol. 259, pp. 271-292, 2015. 
[5] C. Wang and R. P. Agarwal, "Almost periodic dynamics for impulsive delay neural networks of a general type on almost periodic time scales," Communications in Nonlinear Science and Numerical Simulation, vol. 36, pp. 238-251, 2016.

[6] Y.-Y. Yu, L.-L. Wang, and Y.-H. Fan, "Uniform ultimate boundedness of solutions of predator-prey system with MichaelisMENten functional response on time scales," Advances in Difference Equations, Paper No. 319, 14 pages, 2016.

[7] W. S. C. Gurney, S. P. Blythe, and R. M. Nisbet, "Nicholson's blowflies revisited," Nature, vol. 287, no. 5777, pp. 17-21, 1980.

[8] K. Gopalsamy and P. X. Weng, "Feedback regulation of logistic growth," International Journal of Science and Mathematics, vol. 1, pp. 177-192, 1993.

[9] B. S. Lalli, J. S. Yu, and M.-P. Chen, "Feedback regulation of a logistic growth, Dynamic Systems and Applications, vol. 5, no. 1, pp. 117-124, 1996.

[10] L. Liao, "Feedback regulation of a logistic growth with variable coefficients," Journal of Mathematical Analysis and Applications, vol. 259, no. 2, pp. 489-500, 2001.

[11] W.-T. Li and L.-L. Wang, "Existence and global attractivity of positive periodic solutions of functional differential equations with feedback control," Journal of Computational and Applied Mathematics, vol. 180, no. 2, pp. 293-309, 2005.

[12] F. D. Chen, "Permanence of a single species discrete model with feedback control and delay," Applied Mathematics Letters, vol. 20, no. 7, pp. 729-733, 2007.

[13] X. Xie, C. Zhang, X. Chen, and J. Chen, "Almost periodic sequence solution of a discrete Hassell-Varley predator-prey system with feedback control," Applied Mathematics and Computation, vol. 268, pp. 35-51, 2015.

[14] X. Mao, "Stabilization of continuous-time hybrid stochastic differential equations by discrete-time feedback control," Automatica, vol. 49, no. 12, pp. 3677-3681, 2013.

[15] H. A. El-Morshedy and A. Ruiz-Herrera, "Geometric methods of global attraction in systems of delay differential equations," Journal of Differential Equations, vol. 263, no. 9, pp. 5968-5986, 2017.

[16] X. Liu and Y. M. Zeng, "Linear multistep methods for impulsive delay differential equations," Applied Mathematics and Computation, vol. 321, pp. 555-563, 2018.

[17] V. Lakshmikantham, S. Sivasundaram, and B. Kaymakcalan, Dynamic Systems on Measure Chains, Kluwer Academic Publishers, Boston, Mass, USA, 1996.

[18] M. Bohner and A. Peterson, Dynamic Equations on Time Scales: An Introduction with Applications, Birkhauser, Boston, Mass, USA, 2001.

[19] V. L. Kocic and G. Ladas, Global Behavior of Nonlinear Difference Equations of Higher Order with Application, Kluwer Academic, Dordrecht, The Netherlands, 1993.

[20] Y. Fan, Y. Yu, and L. Wang, "Some differential inequalities on time scales and their applications to feedback control systems," Discrete Dynamics in Nature and Society, Art. ID 9195613, 11 pages, 2017. 


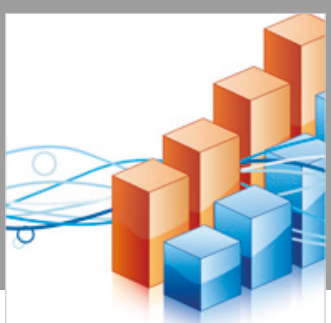

Advances in

Operations Research

\section{-n-m}
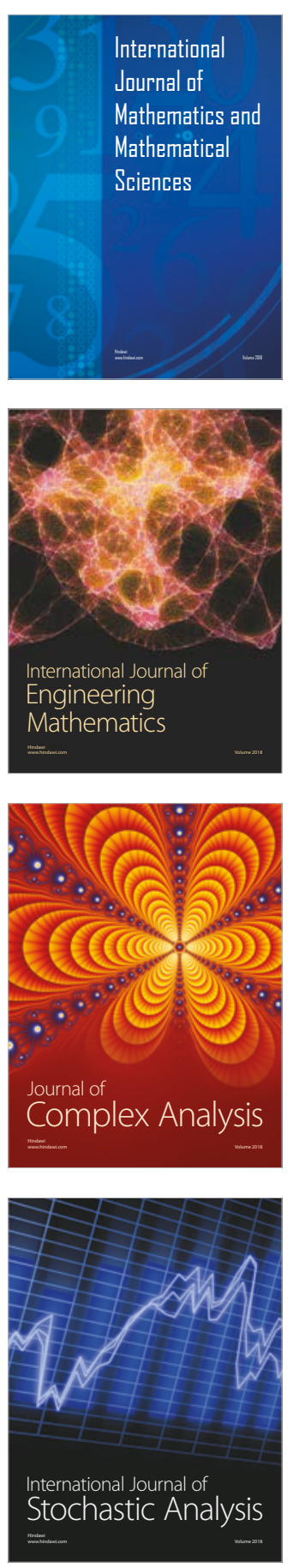
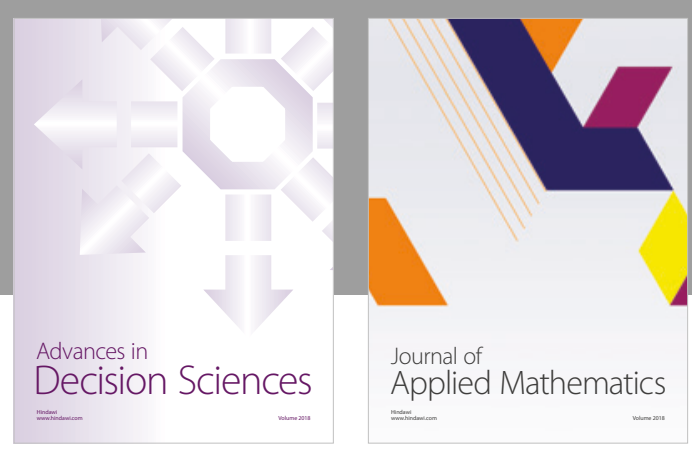

Journal of

Applied Mathematics
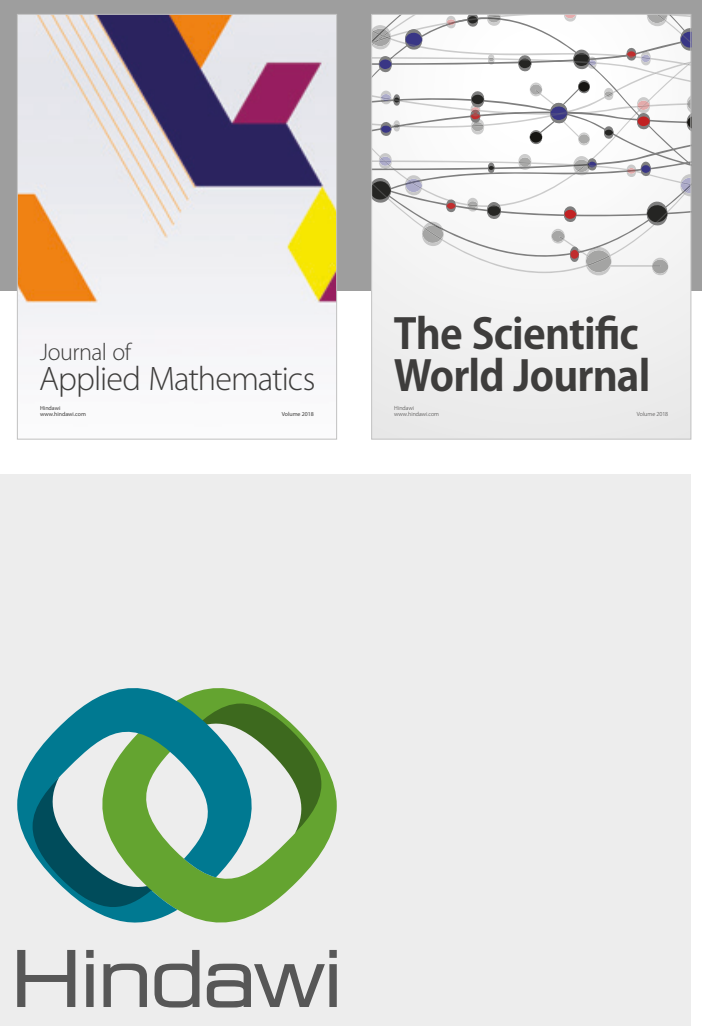

Submit your manuscripts at

www.hindawi.com

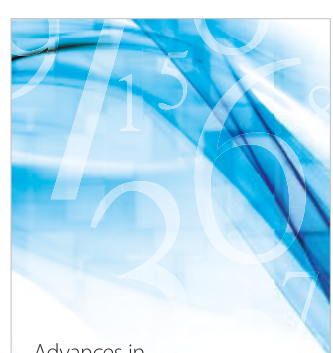

Advances in
Numerical Analysis
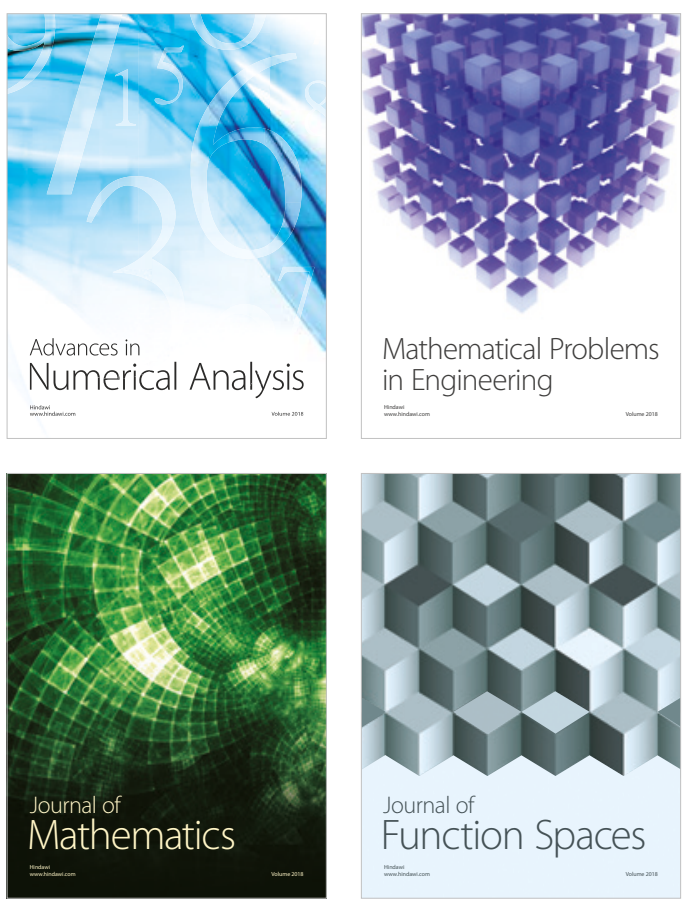

Mathematical Problems in Engineering

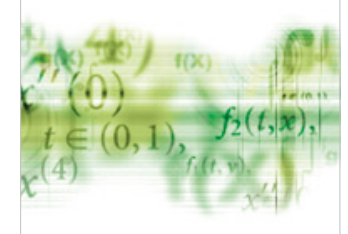

International Journal of

Differential Equations

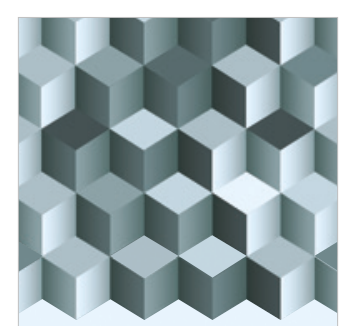

Journal of

Function Spaces

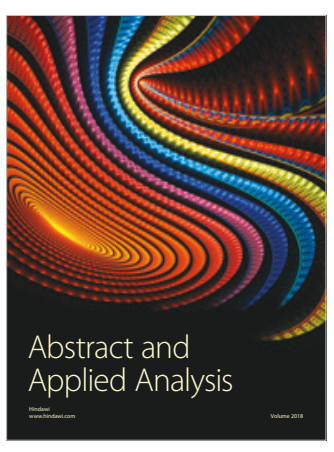

The Scientific

World Journal

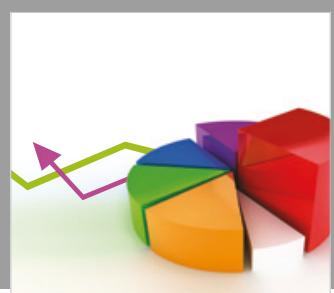

Journal of

Probability and Statistics
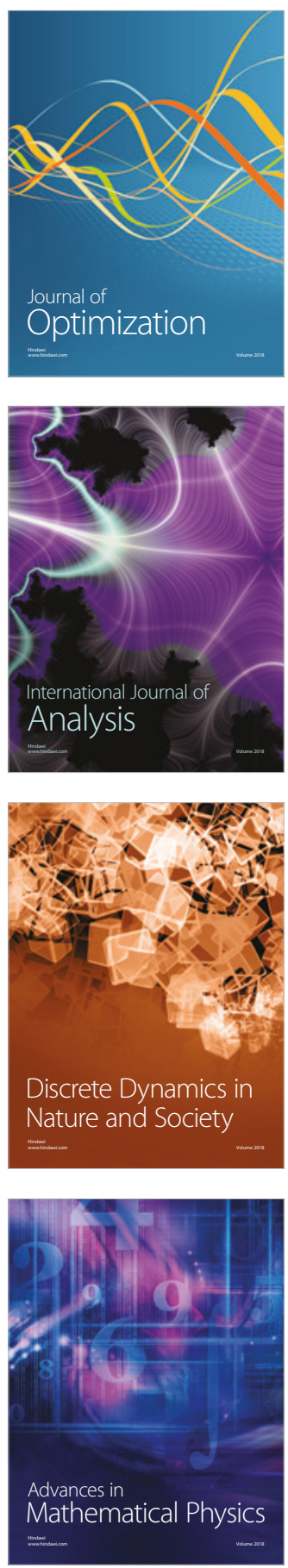